Math. Model. Nat. Phenom.

Vol. 7, No. 3, 2012, pp. 40-48

DOI: $10.1051 / \mathrm{mmnp} / 20127304$

\title{
A Modeling Framework For Immune-related Diseases
}

\author{
F. Castiglione ${ }^{1 *}$, S. Motta $^{2}$, F. Pappalardo ${ }^{2}$, M. Pennisi ${ }^{2}$ \\ ${ }^{1}$ National Research Council of Italy, Rome, Italy \\ ${ }^{2}$ University of Catania, Catania, Italy
}

\begin{abstract}
About twenty five years ago the first discrete mathematical model of the immune system was proposed. It was very simple and stylized. Later, many other computational models have been proposed each one adding a certain level of sophistication and detail to the description of the system. One of these, the Celada-Seiden model published back in 1992, was already mature at its birth, setting apart from the topic-specific nature of the other models. This one was not just a model but rather a framework with which one could implement his own immunological theories.

Here we describe this computational framework, developed to perform simulations of different pathologies that are directly or indirectly connected to the immune system. We briefly describe the system first, then we report on few applications so to give the reader a clear idea of its practical utility in clinical research problems.
\end{abstract}

Keywords and phrases: computational biology, CS-model, immunology, agent-based modeling

Mathematics Subject Classification: 92B05, 46N60, 62P10, 92C60

\section{Introduction}

The complexity of the immune system is very attractive and challenging for those who are experienced in mathematical modeling. The various aspects of the immune system such as recognition, memory, cooperation/competition, localization, variability and heterogeneity are, without questions, captivating themes for theoreticians, no matter their scientific background. Indeed, theoretical immunology has motivated a significant number of researchers coming from disciplines like mathematics, physics, computer science, statistics and chemistry. In the past two or three decades they have developed several mathematical models focused on different aspects of the immune system such as for example immune surveillance, viral infections, complexities of antigen-antibody systems, dynamics of homeostasis and the maintenance of immunological memory, immune suppression and tolerance, and idiotype networks.

The "Theoretical Immunology Workshop", held in 1987 in Santa Fe, was probably the first event which brought together a large number of scientists coming from different disciplines with the aim of gaining understanding of the immune system functioning and regulation [1,2]. Perelson in [3] gives an excellent review of the work done at that time and describes a number of immunological problems in which the use of physical concepts and mathematical methods has increased their understanding.

*Corresponding author. E-mail: f.castiglione@iac.cnr.it 
Whereas the majority of the immune system models are based on differential equations and are generally designed to model specific aspects of the immune response, computer based approaches like the one described here, have shown the potential to describe adequately several immune processes at once. Moreover, it is worth to note that an important drawback of classical mathematical equation-based (i.e., reaction-kinetics) models is the difficulty to exhibit the fluctuations typically seen in immunology [4]. The problem is that while in reaction-kinetics models the information is global, in nature, this is something local. Therefore, modeling approaches that depend on local, rather than global, information are part of a desire to be closer to biology and biological thinking and to be able to make biological approximations rather than mathematical ones [5].

Cellular automata or agent-based models offer a modeling solution that is i) closer to biology and ii) that does not rely on global (averaged) information. A cellular automaton is a discrete dynamical system composed by "cells" located on a regular spatial lattice. A cell has any one of a finite number of states, and it is updated at discrete time intervals based on its prior state and the prior states of its near neighbors. All cells on the lattice are updated synchronously so that the state of the entire system advances in discrete time steps. Agent-based models are generalizations of cellular automata that exploit the individual representation of the entities involved in a process and expand on their description and representation [6].

A spatial process is classically described by a set of partial differential equations with continuous time and space. Cellular automata are the simplest models of spatially distributed processes because each continuous dimension is replaced by a discretized approximation. The goal of automata and agent modeling in immunology is to derive the systems behavior from the interactions between its many constituents. Automata and agent models, unlike equation-based ones, are amenable to experimentation and exploratory tinkering. New automata types (e.g., new cellular components) can be developed incrementally and introduced without affecting other aspects of the model. In a certain sense the "logic of the model" is linear although the combination of simple components often lead to emergent phenomena that are not the sum of the individual contribution. This makes such discrete models fitting perfectly with the aim of the study of complex systems in general.

One of the first attempt to model the immune system with discrete mathematics is the model of Kaufman, Urbain and Thomas introduced back in 1985 [7]. It considered five types of cells represented by a Boolean variable denoting spin up (high concentration) and spin down (low concentration) and simple boolean rules driving its dynamics according to a very qualitative vision of the immune response.

This new theoretical approach has been followed by a number of other studies. However, they all advocated the reductionist approach and represented an immunology very stylized (biologists would likely use the word unfruitful instead). The point to make here is that theoretical models like the aforementioned ones, although useful to describe specific biological mechanisms, are deemed too limited by the clinicians, hence they fail to capture the complexity of any pathology in which the immune system plays a fundamental role. This was the case until a new level of sophistication was set by the model of Celada and Seiden. It is not by chance that this detailed immune system model has been conceived by a reductionistby-nature physicist instructed on the marvels of the immune system by an immunologist that congenitally used to keep all exceptions under the sight when explaining a phenomena. They developed a model more like a modeling platform complex enough to allow the simulation of more processes in immunology.

The IMMSIM automaton is the computer implementation of the model of Celada and Seiden. This can be also seen as "model of immunological models" [8-11]. The author's goal was to capture as much as possible of the dynamics of the immune system and to be able to make experiments in machina. In so doing, they assembled a number of up-to-date immunological theories in one single coherent and generalpurpose model that has shown to be a valid starting point to explore different medical/clinical subjects. In the past years we have developed specialized versions of the Celada-Seiden model to study HIV-1 infection, EBV infection, hypersensitivity reactions and cancer immunoprevention (described respectively in $[12-15])$, showing that the platform provides enough complexity to be able to implement processes 
relative to the immune system activity and related to different clinical applications. This constitutes the content of the following section.

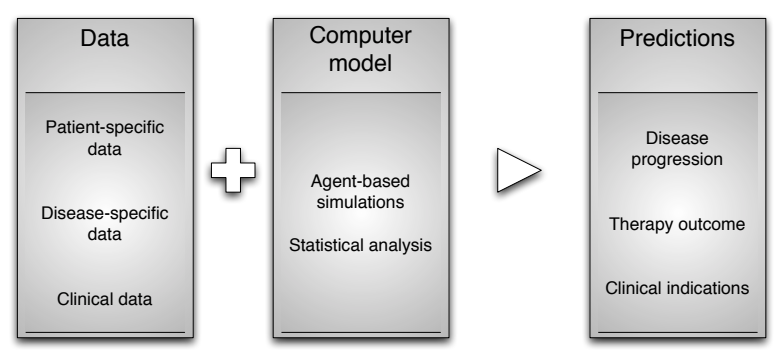

FigURE 1. The aim of the presented simulation platform is to provide enough complexity to be able to implement virtually any process in immunology.

\section{Applications}

C-IMmSim, a reimplementation of the IMMSIM automaton in C language, has been employed in the study of different clinical setting to describe a few pathological conditions. The model accommodates inputs as clinical data, disease specific data and patient-specific. The statistically derived output of the simulations gives predictions not only on the disease progression but also clinical indications like the outcome of an hypothetical therapeutic regimen or a new therapeutic mechanism (see Fig. 1).

We now describe different applications of C-IMMSim relative to i) infectious diseases like Epstein-Barr virus and HIV, ii) allergies and iii) atherosclerosis. The treatment is purposefully kept at a simple level, sending the reader to the references for further readings.

\subsection{Modeling infectious diseases}

One of the first application of the present architecture has been to model infectious diseases and in particular the HIV-1 infection [12] and the effects of antiretroviral therapy [16]. In the model (that follows the original idea of [17]), bit strings represent binding sites of cells and the virus. The model includes the major classes of cells of the lymphoid lineage ( $\mathrm{T}$ helper lymphocytes, cytotoxic $\mathrm{T}$ lymphocytes, B lymphocytes, antibody-producer plasma cells and natural killers) and some of the myeloid lineage (macrophages and dendritic cells). In line with the agent-based paradigm, these entities are individually represented. In contrast to cells, cytokines like interleukin- 2 are represented in terms of concentrations and their dynamics described by a parabolic partial differential equation plus a degradation term accounting for the finite half-life $[18,19]$. Modeling features of the HIV infection include HIV replication inside infected lymphocytes, T production impairment; specific response against HIV strains and HIV mutation. In particular, the HIV has been implemented by a life cycle specified by the following stages: i) the virus infects $\mathrm{CD} 4^{+} \mathrm{T}$ cells, macrophages, dendritic cells; ii) reverse transcriptase copies the viral single stranded RNA genome into a double-stranded viral DNA. The viral DNA is then integrated into the host chromosomal DNA; iii) the virus remains at rest until an event activates the transcription; iv) the replicating virus buds from the cell membrane. Fully assembled virions are then able to infect other cells to restart the life cycle. The effect of the therapy (Higly Active AntiRetroviral Therapy $=$ HAART) are modeled as follows: Reverse Transcriptase inhibitors (RTI) block reverse transcriptase enzymatic functions and disrupt completion of synthesis of the double-stranded viral DNA thus preventing HIV-1 from replicating (i.e., it prevents the virus in stage i) from reaching stage ii); Protease Inhibitors (PI) prevent viral replication by inhibiting the activity of HIV-1 protease, an enzyme used by the virus to 
cleave nascent proteins for final assembly of new virions (i.e., it prevents virus assembly in stage iv). For what concerns the setting of the parameters related to the therapy, computer simulations have been performed in which the immunological parameters at the time of therapy initiation on the basis of the average values measured in patients in vivo we kept fixed: $5.8 \pm 0.2 \mathrm{RNA}$ copies $/ \mathrm{ml}$ (in logarithmic scale), $870 \pm 50 \mathrm{CD} 4$ cells $/ \mu l$ and $430 \pm 50 \mathrm{CD} 8$ cells $/ \mu l$. For all simulations we applied a one-year course of HAART.

Simulation results, in line with a number of studies, indicate that interfering with HIV replication by starting the therapy in the early phases of the infection could have a deep impact on the whole disease course. Indeed, we found that an opportunity time-window exists for the initiation of HAART (roughly within three weeks before the establishment of viral reservoirs), in which the therapy can control viral replication, preventing generalized immune activation and extensive CD4+ T cell depletion $[16,20,21]$

Another application, still relating to infectious diseases, has been to model the infection of the EpsteinBarr virus (EBV). The EBV infects more than $90 \%$ of humans benignly for life but, unfortunately, it can be associated with tumors. It is a uniquely human pathogen that is amenable to quantitative analysis; however, there is no applicable animal model. In this respect, computer models may provide a virtual environment to perform experiments not possible in human volunteers. This is the reason why we applied a fairly sophisticated stochastic cellular automaton to the modeling of EBV infection. The implementation of EBV simulation included new parameters/processes with respect to the core model: i) The probability that a naive B cell encountering a virus makes the transition to the latent state within the virtual lymph node is set to near unity; ii) The probability that a latent B cell becomes lytic is based on values in [22]; iii) The viral growth inside a lytic $\mathrm{B}$ follows an exponential growth $e^{m t}$ with $m=0.35$; iv) A lytic $\mathrm{B}$ cell that is filled with newly assembled viruses bursts when the number of viruses reaches $10^{3}$. Of these, just $5 \%$ are considered infectious to account for the fact that most virus is trapped on the surface of nearby cells, yet only one virus is needed to start an infection; v) The growth rate $m$ and the burst size are chosen to fix at one day on average, the time it takes a lytic B cell to burst once it starts viral replication; vi) Latently infected $B$ cells arrive from the blood compartment at about one cell every three days on average. This rate is lower than the actual estimated return rate.

The results show that infected B-cell dynamics in the acute and chronic phases of infection correspond well to clinical data including the establishment of a long term persistent infection (up to 10 years) that is absolutely dependent on access of latently infected B cells to the peripheral pool where they are not subject to immunosurveillance. The most important outcome of this study was that in the absence of this compartment the infection is cleared [13].

\subsection{Modeling hypersensitivity}

A quite different implementation of the model has been the one able to simulate hypersensitivity reactions (i.e., allergies like asthma). In medical term a patient suffering some form of allergy is called atopic. For reasons that are not yet understood, these people have a predisposition to respond to some environmental antigens (e.g., pollen, mold spores) by producing antibodies of the IgE class. Since this trait tends to run in families, it probably has a genetic component. It is estimated that over $30 \%$ of the world population is atopic. Moreover, the number of people suffering from atopic diseases is increasing in the industrialized countries, revealing a link between modern style of life and atopy ([23]).

In recent decades, scientists, clinicians and epidemiologists have elucidated the intra-cellular and the cellular mechanisms involved in allergic reactions, including the roles of TH helper subsets and interleukins ([24]). However, our understanding is still lacking as to the full sequence of events involved in disease development, and to the key factors determining the differences between a person who is allergic to, say, grass pollen and one who is allergic to bee venom. The only agreement seems to be that allergenicity is a consequence of a complex series of interactions involving, not only the allergen, but also the dose, the sensitizing route, sometimes an adjuvant, and most importantly, the genetic constitution of the recipient $([23,25,26])$. 
Acute or immediate type I hyper-sensitivity reaction is a consequence of mediators (histamine, leukotrienes, prostaglandin etc.) released by mast cells (MC) or basophils triggered via the allergenmediated cross-linking of cell surface bond immunoglobulin-E ( $\mathrm{IgE})$. Convincing evidence has accumulated, suggesting that the immune response to allergens in atopics is biased towards the $\mathrm{T}$ helper type II (TH2) phenotype, characterized by the production of the interleukin-4 (IL-4) and interleukin-5 (IL-5). These are key cytokines in class switching to IgE (replacing IgG) in B cells and in the accumulation and activation of eosinophils respectively ([24]). Allergic IgE responses occur mainly on mucous membrane surfaces in response to allergens (i.e., common environmental antigen), which enter the body either by inhalation or ingestion. Typically, such responses manifest themselves in systemic anaphylaxis, localized or hay fever, asthma, hives, food allergies and eczema. Most allergens are small proteins, or protein-bound substances, having a low molecular weight. Common antigens, associated with type I hyper-sensitive reactions are proteins, such as foreign sera or vaccines, drugs, such as penicillin or sulfonamides, local anesthetics (most drugs are low-molecular weight compounds that are incapable of inducing immune responses, unless conjugated with a larger molecule; these small molecules first react with proteins which work as hapten-carriers to form drug-proteins derivatives) etc.(see [24]).

In [27] we described the development of a simulation tool for the gene regulatory network for the switch of $\mathrm{TH}$ cells and how we integrate it in the agent-based mesoscopic simulator of type I allergic response. The work is one of the first attempts to integrate two mathematical/computational descriptions of the mesoscopic cellular level of the immune system functions (lymphocyte interaction, antigen presentation, humoral response, etc.) with a microscopic gene-network dynamics represented by a Boolean-type dynamical network. Also in this case we were able to show that the resulting dynamics of the system is consistent with biological knowledge.

In particular the multi-level simulator reproduced the hallmarks of an IgE-mediated hypersensitive reaction and ultimately provided an example of how to combine the mesoscopic level description of immune cells with the microscopic gene-level dynamics.

\subsection{Modeling atherosclerosis}

Atherosclerosis, a pathology affecting arterial blood vessels, is one of most common diseases of developed countries. It is, in large part, due to the deposition of low density lipoproteins (LDL), i.e., plasma proteins carrying cholesterol and triglycerides that determine the formation of multiple plaques within the arteries [28].

The origin of atherosclerosis is still not fully understood. However there are risk factors that increase the probability of developing atherosclerosis in humans. Some of these are within the individual control (smoking, obesity), while others seem to have a genetic origin (familial hypercholesterolemia, diabetes, hypertension) [29].

The common denominator in all forms of atherosclerosis is the elevated level of LDL, which is subject to oxidation, becoming oxidized LDL (oxLDL) that promotes inflammatory response and immune system activation in the artery walls [30]. The formation of atherosclerotic plaques in the artery reduces both the internal diameter of vessels and the blood flux, leading to a number of serious pathologies. Atherosclerosis and their anatomical consequences cause severe problems. Stenosis (narrowing) and aneurysm of the artery are chronic, progress slowly, and are the cumulative effects indicative of the progression of atherosclerotic disease. In both cases the result is an insufficient blood supply to the organ fed by the artery. Most commonly, soft plaque suddenly ruptures, causes the formation of a thrombus that will rapidly slow or stop blood flow, leading to death of the tissues fed by the artery. This catastrophic event is called infarction and is not predictable. The most common event is thrombosis of the coronary artery causing infarction (a heart attack). However, since atherosclerosis is a body-wide process, similar events also occur in the arteries of the brain (stroke attack), intestines, kidneys, etc. Those atherosclerosis-associated events are often the cause of death or serious invalidate diseases and require preventive treatments. 
As atherosclerosis is recognized as an immune system-related pathology, vaccine research in this field represents a hot pharmaceutical topic. Recently we proposed a model based on the agent-based paradigm [31] and belonging to the aforementioned simulation framework, which reproduces clinical and laboratory parameters associated to atherogenesis. The model and its computer implementation (called SimAthero) consider all the relevant variables that play an important role in atherogenesis and its induced immune response, i.e. LDL, oxLDL, OLAB, chitotriosidase, and the foam cells generated in the artery wall.

SimAthero contributes by simplifying the influence of each component in the pathogenesis of atherosclerosis and applies to the very early stages of the atherosclerosis, i.e., before a calcified plaque is formed. In silico experiments on two samples of one hundred virtual humans show reasonable agreements with clinical observations. The model and its computer implementation are very flexible and new biological entities and interactions can be easily added. The motivation that pushes us in developing such a computational model is the need to better understand the intriguing network of interactions among immune system entities in the pathogenesis of atherosclerosis. Much work has been done trying to explain the individual role of different immune system players. As the immune system can be considered as complex intelligent system that emerges from independent not-centralized behavior, an agent-based model that tries to capture this emergent behavior is helpful.

Presently the model is very naive when compared to real and complex biological dynamics of the atherogenesis, but it can be used as a starting point to evaluate some theoretical hypothesis that cannot be tried directly in clinical trials. For example, the model produced an important suggestion for future biological experiments on the role of OLAB in the activation of the macrophage system to clear the vessels as observed in thalassemic patients where the LDL level is low and OLAB concentration is elevated. Actually we use the model to simulate the behavior of patients with familial hypercholesterolemia, due to the absence of LDL receptor in macrophages, which is characterized by an enormous generation of foam cells and severe atherosclerotic lesions. In this situation the model would be useful to control the effect of intensive LDL-reducing treatments (i.e. plasmapheresis) and high dosage statin treatments. In this framework, if SimAthero simulations predictions will be experimentally validated, it will be possible to obtain important information on the duration of treatment and their frequency.

\section{Similar or related approaches}

The central idea of the present article is to have a general model of the immune system and to use it to perform different studies. The immune system is unique, hence the model should be one incorporating all aspects of previous studies so to have an incremental complexity of the model rather than a tailored and disposable use of models. This approach has recently been followed by others. Herein we report the most known approaches.

The first named Simmune $[5,32]$ attempts to produce more a modeling technique or language than a specific model. This agent simulation framework and environment can be used to develop models of the immune system. It is based on a model of particle interactions that can be used to create detailed models of the immune system. Simmune simulates interactions between cells and molecules as agents and concentrations of agents on a grid of arbitrary granularity. The abstract physics of the framework are described as follows. Simmune is a tool to simulate interacting particles that have a continuous spectrum of states. Transitions between the states are probabilistic events triggered by the exchange of messenger particles with limited ranges. Simulations are done in discretized space-time: The particles are living on a grid and their states are (asynchronously) updated at discrete time steps. Transitions between the particle states depend on the messenger field intensities, typically involving a threshold field intensity. The messenger field intensities are calculated by integration of reaction-diffusion equations [32]. A major advantage of Simmune is that it allows models that combine direct intercellular interactions (such as those between an antigen and a B-cell) and indirect communication via molecular messengers (such as lymphokines). It also supports spatial compartmentalization and communication conduits. Overall, it provides a framework and simulation environment that supports very detailed models of the immune system. 
A second approach attempts to include all possible details of the immune system and to model the dynamics, using either various mathematical methods, such as Monte Carlo simulations is Immunosim [33]. This model attempts to provide a complete simulation of the spatially extended dynamics in the thymus and to study selection using the simulation [33]. The advantage of these general simulations is that they include all details of the current knowledge. Their disadvantage is their complexity that does not allow us to understand in details the reason for the observed dynamics, and their sensitivity to parameter changes is a drawback common to many agent-based models of the immune system.

In [34] the authors have developed a software system called Simissys that models and simulates aspects of the human immune system based on the computational framework of cellular automata. We model tens of thousands of cells as exemples of the significant players in the functioning of the immune system, and simulate normal and simple disease situations by interpreting interactions among the cells. The current version of Simisys models and simulates the innate and adaptive components of the immune system. The specific players we model are the macrophages, dendritic cells, neutrophils, natural killer cells, B cells, T helper cells, complement proteins and pathogenic bacteria.

Another approach is given in [35]. The Basic Immune Simulator (BIS), an agent-based model created to study the interactions between the cells of the innate and adaptive immune system. The BIS simulates basic cell types, mediators and antibodies, and consists of three virtual spaces representing parenchymal tissue, secondary lymphoid tissue and the lymphatic/humoral circulation. The BIS includes a graphical user interface to facilitate its use as an educational and research tool. The BIS was used to qualitatively examine the innate and adaptive interactions of the immune response to a viral infection.

\section{Discussion and conclusions}

In about twenty five year of computational immunology a number of mathematical and computational models of the immune system and its interaction with pathogens of all sorts have been developed. Most of them are equation based. Others belong to the class of discrete models known as cellular automata or agent-based. This latter modeling paradigm gives the great advantage to build large and scalable models that should be conceived more like developing platforms rather than models.

In this article we have described the pioneer of this class of models, the Celada-Seiden model, and the way it has fostered the development of tailored solutions to specific medical/clinical studies. After years, this approach remains successful as witnessed by other attempt to build general purpose modeling frameworks in immunology. However, a note of warning should be given at this point since it seems that there are no drawbacks in using such models. Indeed there is a major flip of the coin when compared to equation based models, consisting in the greater difficulty in estimating parameters from data. Parameter estimation can be done but at the cost of a considerable number of simulations(agent-based models require considerable amount of processing power but, luckily, the technological advances in semiconductor industry alleviate this problem). Secondly, a lack of mathematical formalism makes difficult the communication of complicated models, hampering it widespread adoption in the scientific community. Moreover, without a formal description, it is difficult to formally analyze complex agent-based systems. While computer simulations can be very useful, results obtained through simulations do not formally validate the observed behavior. In conclusion, there is a need for a mathematical framework which one can use to represent agent-based models and formally establish their properties. Attempt to focus on this problem and to use the framework of finite dynamical systems is given in [36]. There, both, deterministic and stochastic versions of this framework are discussed. The paper also contains a sampling of the mathematical results from the literature to show how finite dynamical systems can be used to carry out a rigorous study of the properties of agent-based systems and also show how the framework can also serve as a universal model for computation.

A final note goes on the possibility to develop large-scale epidemiological models like EpiSimS [37]. EpiSimS implements a spatial epidemic model based on realistic geographical maps and integrates disease models with patterns of interpersonal human contact [38]. Along this line, the model of the immune system described in this article fit in with the expansion of the epidemiological model (like EpiSimS) 
where the infectivity of a single individual modeled at the epidemiological level, can be determined by running a single immune response simulation at the individual level. The so-constructed model would be truly multi-scale, possibly allowing healthcare organizations to make more accurate predictions of the spread of epidemics in this era of high mobility.

\section{References}

[1] A.S. Perelson, G. Weisbuch (Eds.). Theoretical Immunology, Part One. Springer, Berlin, 1988.

[2] A.S. Perelson (Ed.). Theoretical Immunology, Part Two. Addison Wesley, Redwood City, CA, 1988.

[3] A.S. Perelson, G. Weisbuch. Immunology for physicists. Rev. Mod. Phys., 69 (1997), 1219-1267.

[4] R. Puzone, B. Kohler, P.E. Seiden, F. Celada. IMMSIM, a flexible model for in machina experiments on immune system responses. Future Gener. Comput. Syst., 18 (2002), No. 7, 961-972.

[5] M. Meier-Schellersheim, G. Mack. Simmune: A tool for simulating and analyzing immune system behavior. Das Deutsche Elektronen-Synchrotron Technical Report, 1999.

[6] F. Castiglione Agent based modeling. Scholarpedia, 1 (2006), No. 10, 1562.

[7] M. Kaufman, J. Urbain, R. Thomas. Towards a logical analysis of the immune response. J. Th. Biol., 114 (1985), $527-561$.

[8] F. Celada, P.E. Seiden. Affinity maturation and hypermutation in a simulation of the humoral immune response. Eur. J. Immunol., 26 (1996), No. 6, 1350-1358.

[9] F. Celada, P.E. Seiden. A computer model of cellular interactions in the immune system. Immunol. Today, 13 (1992), No. 2, 56-62.

[10] P.E. Seiden, F. Celada. A model for simulating cognate recognition and response in the immune system. J. Th. Biol., 158 (1992), No. 3, 329-357.

[11] D. Morpurgo, R. Serenthà, P.E. Seiden, F. Celada. Modelling thymic functions in a cellular automaton. Int. Immunol., 7 (1995), No. 4, 505-516.

[12] F. Castiglione, F. Poccia, G. D’Offizi, M. Bernaschi. Mutation, fitness, viral diversity and predictive markers of disease p rogression in a computational model of HIV-1 infection. AIDS Res. Human Retrovirus, 20 (2004), No. 12, 1316-1325.

[13] F. Castiglione, K.A. Duca, A.S. Jarrah, R. Laubenbacher, K. Luzuriaga, D. Hochberg, D.A. Thorley-Lawson. Simulating Epstein-Barr virus infection with C-ImmSim. Bioinformatics, 23 (2007), 1371-1377.

[14] F. Castiglione, V. Sleitser, Z. Agur. Analyzing hypersensitivity to chemotherapy in a Cellular Automata model of the immune system, in Cancer Modeling and Simulation, L. Preziosi (Ed.), pages 333-365. Chapman \& Hall/CRC Press, London, UK, 2003.

[15] F. Pappalardo, P-L. Lollini, F. Castiglione, S. Motta. Modelling and simulation of cancer immunoprevention vaccine. Bioinformatics, 21 (2005), No. 12, 2891-2897.

[16] P. Paci, R. Carello, M. Bernaschi, G. D'Offizi, F. Castiglione. Immune control of HIV-1 infection after therapy interruption: immediate versus deferred antiretroviral therapy. BMC Infect. Dis., 9 (2009), 172.

[17] J.D. Farmer, N.H. Packard, and A.S. Perelson. The immune system, adaptation and machine learning. Physica D, 22 (1986), 187-204.

[18] P. Paci, F. Castiglione, M. Bernaschi, V. Baldazzi. A discrete/continuous model of anti-HIV response and therapy. IEEE Computer Society, Digital library Proceedins UKSIM, pages 481-486, 2008.

[19] V. Baldazzi, P. Paci, M. Bernaschi, F. Castiglione. Modeling lymphocytes homing and encounters in lymph nodes. BMC Bioinformatics, 10 (2009), 387.

[20] P. Paci, F. Martini, M. Bernaschi, F. D'Offizi, F. Castiglione. Earlier is better: a timely HAART initiation may pave the way for best controllers. BMC Infectious Diseases, 11 (2011), 56.

[21] F. Castiglione, P. Paci. Criticality of timing for anti-HIV therapy initiation. PLoS ONE, 5 (2010), No. 12, e15294.

[22] L.L. Laichalk, D.A. Thorley-Lawson. Terminal differentiation into plasma cells initiates the replicative cycle of EpsteinBarr virus in vivo. J. Virol., 79 (2005), 1296-1307.

[23] A.B. Kay AB. Allergy and allergic diseases. First Part. N. Engl. J. Med., 344 (2001), 30-37.

[24] R.A. Goldsby, T.J. Kindt, B.A. Osborne. Kuby Immunology, IV ed.. W.H. Freeman \& Co., NY, 2000.

[25] A.B. Kay. Allergy and allergic diseases. Second Part. N. Engl. J. Med., 344 (2001), 109-113.

[26] S.T. Holgate. Allergic disorders. British Med. J., 320 (2000), 231-234.

[27] D. Santoni, M. Pedicini, F. Castiglione. Implementation of a regulatory gene network to simulate the TH1/2 differentiation in an agent-based model of hyper-sensitivity reactions. Bioinformatics, 24 (2008), 1374-1380.

[28] G.K. Hansson. Inflammation, atherosclerosis, and coronary artery disease. N. Engl. J. Med., 352 (2002), No. 16, 1685-1695.

[29] A. Romero-Corral, V.K. Somers, J. Korinek, J. Sierra-Johnson, R.J. Thomas, T.G. Allison, F. Lopez-Jimenez. Update in prevention of atherosclerotic heart disease: management of major cardiovascular risk factors. Rev. Invest. Clin., 58 (2006), No. 3, 237-244.

[30] C. Weber, A. Zernecke, P. Libby. The multifaceted contributions of leukocyte subsets to atherosclerosis: lessons from mouse models. Nat. Rev. Immunol., 8 (2008), No. 10, 802-815.

[31] F. Pappalardo, S. Musumeci, S. Motta. Modeling immune system control of atherogenesis. Bioinformatics, 24 (2008), No. $15,1715-1721$. 
[32] M. Meier-Schellersheim. The immune system as a complex system: Description and simulation of the interactions of its constituents. Ph.D. thesis, University of Hamburg, Germany, 2001.

[33] S. Efroni, D. Harel, I.R. Cohen. Toward rigorous comprehension of biological complexity: modeling, execution, and visualization of thymic T-cell maturation. Genome Res., 13 (2003), No. 11, 2485-2497.

[34] J.K. Kalita, K. Chandrashekar, R. Hans, R. Selvam. Computational modelling and simulation of the immune system. Int. J. Bioinf. Res. Appl., 2 (2006), No. 1, 63-88.

[35] V.A. Folcik, G.C. An, C.G. Orosz. The basic immune simulator: an agent-based model to study the interactions between innate and adaptive immunity. Theor. Biol. Med. Model., 4 (2007), 39.

[36] R.C. Laubenbacher, A.S. Jarrah, H.S. Mortveit, S.S. Ravi. A mathematical formalism for agent-based modeling. CoRR., (2008), abs/0801.0249.

[37] S.Y. Del Valle, P.D. Stroud, J.P. Smith, S.M. Mniszewski, J.M. Riese, S.J. Sydoriak, D.A. Kubicek. Episims: Epidemic simulation system. Los Alamos Unlimited Release (LAUR), (2006), 06-06714.

[38] S. Mniszewski, S.Y. Del Valle, P. Stroud, J. Riese, S. Sydoriak. Episims simulation of a multi-component strategy for pandemic influenza. Proceedings of the 2008 Spring Simulation Multiconference, 2008. 